\title{
Retraction Note: Excavation and support method of tunnel with high ground stress and weak surrounding rock based on GIS
}

\author{
Zhichun Fang $^{1,2} \cdot$ Zhengguo Zhu $^{1,2,3} \cdot$ Pengfei Wu ${ }^{4} \cdot$ Renyuan Wang ${ }^{1} \cdot$ Chaoyi Ma ${ }^{1} \cdot$ Zhiwei Wang $^{2}$ \\ Published online: 15 December 2021 \\ (c) Saudi Society for Geosciences 2021
}

Retraction Note: Arabian Journal of Geosciences (2021) 14: 537

https://doi.org/10.1007/s12517-021-06747-7

The Editor-in-Chief and the Publisher have retracted this article because the content of this article is nonsensical. The peer review process was not carried out in accordance with the Publisher's peer review policy. Authors Zhichun Fang, Zhengguo Zhu and Zhiwei Wang have not responded to correspondence regarding this retraction. The Publisher has not been able to obtain a current email address for authors Pengfei Wu, Renyuan Wang and Chaoyi Ma.

The original article can be found online at https://doi.org/10.1007/ s12517-021-06747-7.

Zhengguo Zhu

zzgstd@126.com

1 State Key Laboratory of Mechanical Behavior and System Safety of Traffic Engineering Structures, Shijiazhuag Tiedao University, Shaoxing 050043, Hebei, China

2 State Key Laboratory for Track Technology of High-Speed Railway, China Academy of Railway Sciences, Beijing 100081, China

3 Hebei Province Technical Innovation Center of Safe and Effective Mining of Metal Mines, Shijiazhuang 050043, Hebei, China

4 China Nuclear Power Engineering, Shijiazhuang 050043, Hebei, China 